\title{
Ovarian cancer health politics in Romania and Germany: A comparative study
}

\author{
IONUT MARCEL COBEC ${ }^{1}$, IOAN SAS $^{2}$, AURICA ELISABETA MOATAR $^{3,4}$, \\ LAVINIA MOLERIU $^{5}$ and ANDREAS REMPEN ${ }^{1}$
}

\begin{abstract}
${ }^{1}$ Clinic of Obstetrics and Gynecology, Diakonie-Klinikum Schwäbisch Hall, D-74523 Schwäbisch Hall, Germany;
${ }^{2}$ Department of Obstetrics and Gynecology, 'Victor Babeș' University of Medicine and Pharmacy of Timisoara, 300041 Timisoara, Romania; ${ }^{3}$ Clinic of Internal Medicine, Hohenloher Krankenhaus Öhringen, D-74613 Öhringen, Germany; Departments of ${ }^{4}$ Pharmacology and Biochemistry, and ${ }^{5}$ Medical Informatics and Biostatistics, 'Victor Babeș' University of Medicine and Pharmacy of Timisoara, 300041 Timisoara, Romania
\end{abstract}

Received June 14, 2021; Accepted July 14, 2021

DOI: 10.3892/etm.2021.10651

\begin{abstract}
Ovarian cancer is known to have a poor prognosis, being the 7th most common cancer type in women with regard to incidence and mortality worldwide. The present study underlines the importance of early diagnosis and prevention management of ovarian cancer. This study is a hospital-based case-control study that was conducted at the same time in two similar hospitals from different countries, Romania and Germany, over three years. The results showed that significant differences were identified for the two groups with regards to the age factor $(\mathrm{P}<0.001)$. A risk analysis was performed to determine whether the patients from Romania were exposed to a risk factor. The risk of developing deadly diseases was deemed much higher owing to insufficient protocols or informative programs in Romania. The medical information, early diagnosis and standardized therapy with optimum treatment based on health policy and health care systems, play a key role concerning the management and prognosis of ovarian cancer which are different from country to country.
\end{abstract}

\section{Introduction}

Ovarian cancer has a global incidence of 42 cases per 100,000 and is the second cause of death among the gynecological cancers and the 7th cause of death among women (1-6). Having the highest mortality rate means it is considered a 'silent killer' (2,7-13).

Correspondence to: Dr Ionut Marcel Cobec, Clinic of Obstetrics and Gynecology, Diakonie-Klinikum Schwäbisch Hall, 10 Diakoniestrasse, D-74523 Schwäbisch Hall, Germany

E-mail: cobec_i@yahoo.com

Key words: ovarian cancer, Romania, Germany, early diagnosis, standardized therapy, gynecological cancer
Although ovarian cancer is a worldwide public health issue, there is currently no safe method to diagnose this pathology and form of prevention or early diagnosis of ovarian cancer are very rare. This cancer type is usually diagnosed by routine medical check-up through ultrasound indicating suspected ovarian cancer, or the patient requests a medical examination because of clinical signs including abdominal pain or ascites with clinical relevance (7,9,13-15).

The etiology and pathology of ovarian cancer are not fully known $(5,8,11,16,17)$. The ovarian cancer pathology includes angiogenesis, hematopoiesis, hemodynamic and vascular homeostasis (the production of ascites) mediated by vascular endothelial growth factor (VEGF), a target molecule for peritoneal metastasis (4). Cancer pathology involves not only the functions but also the immune system. Angiogenesis, hypoxia and inflammation can be identified on the cellular level (18). Excessive angiogenesis is mainly a feature of malignant development, but also an important component for other pathologic conditions (4,19-25).

The aim of the present study was to underline the importance of early diagnosis. The study was conducted in similar situations in two different countries. Although in Romania the incidence of ovarian cancer, according to official data, is among the lowest in central and southeast Europe the mortality is extremely high (26). According to the World Health Organization (WHO), and The International Agency for Research on Cancer for Romania, the estimated incidence of ovarian was 13.6 [age-standardized mortality rate (in Europe) per 100,000] and the average mortality rate was 7.0 (27). In the same report concerning ovarian cancer official data in Germany it was reported that the estimated incidence had a rate of 10.2 [age-standardized mortality rate (in Europe) per 100,000] and the average mortality rate was $7.0(27,28)$. Based on this official information a similar mortality was observed, although the incidence is different. In the present study, the situation at two different geographical points of Europe (Germany/Romania), but in similar hospitals, was analyzed with ovarian cancer being the common denominator. 
The majority of studies and guidelines have shown that patient age and stage of the disease play a key role in the prognosis $(29,30)$. On the other hand, access to therapy according to the guidelines, having protocols or informative programs is beneficial to the patients and increases the surveillance (31).

\section{Patients and methods}

Patient data. This is a hospital-based case-control study that was conducted over three years (January 2011-December 2013) in the same period of time in two similar hospitals in Romania and Germany. The study contains data that have been conducted and processed individually. The data were collected in the Clinic of Obstetrics and Gynecology of the Diakonie Klinikum, Schwäbisch Hall, Germany, and in the Municipal Clinical Emergency Hospital of Timisoara, Romania. This study used pre-existing, anonymized and irreversibly de-identified data. Approval from the ethics committee was not required.

Variables. Newly diagnosed cases and those under regular medical treatment with a known malignant ovarian pathology were included. The German database included 49 patients with malignant ovarian pathology and the information was registered according to patient age, tumor type, tumor size, degree of spread to regional lymph nodes, distant metastasis (TNM), grading, and FIGO classification for staging of ovarian cancer as determined by the International Federation of Gynecology and Obstetrics (FIGO). Comparatively the registered data from Romania regarding the age, anatomopathological diagnosis and the TNM classification were according to the described ovarian pathology. There were 97 malignant cases in the Romanian database.

Data were registered from patients at stages 1 to 3 to underline the importance of early diagnosis and prevention management of ovarian cancer.

Statistical analysis. For the statistical analysis we used the following software, respectively, SPSS17.0, Epi Info7 and Microsoft Excel. Descriptive statistics was realized by calculating the mean values, the dispersion parameters, as well as the frequency tables. For the statistical significance non-parametrical tests were conducted in order to determine whether there were any statistical differences in the management of the disease for the two countries (Chi-square test of proportions). For the entire study the significance threshold of $\alpha=0.05$ (5\%) corresponding to the 95 confidence interval (CI) was used. The highlighting of the observed significant differences was realized through calculation of the P-value. For the risk analysis the odds ratio (OR) parameter, and the $95 \%$ CI were calculated; for statistical significance the Chi-square test was applied.

\section{Results}

Age and disease development. Regarding the age between the two groups, there were high differences $(\mathrm{P}<0.001)$, as indicated in Table I. The differences between the two countries regarding the development and the dynamic of this disease are presented in Table II. For stage 1 there are no significant differences, but for the more advanced stages there was a significant increase among the Romanian population.
Risk analysis. Owing to the significant differences between the two groups, a risk analysis was performed to determine whether the patients from Romania were exposed to a risk factor. In addition, since in Romania there are not as many protocols or informative programs, the risk of developing lethal diseases is much higher. In Romania there are two main problems, i.e., patients who come to the doctors in their final stage or cases where the used protocols are not up to date, meaning that the patient easily 'slips' from stage 1 into stage 3 (stage 3 vs. stage 2: $\mathrm{P}<0.001, \mathrm{OR}=6.87,95 \% \mathrm{CI}=2.29-20.61$ or stage 3 vs. stage $1+2: \mathrm{P}=0.026, \mathrm{OR}=2.45,95 \% \mathrm{CI}=1.10-5.47)$. All the results are presented in Table III.

\section{Discussion}

This study presented the situation of ovarian cancer in two regional hospitals in Romania and Germany. Regarding the age of our patients it was determine that between the two groups there were significant differences $(\mathrm{P}<0.001)$. According to the official data from the German Cancer Register, the median age at which patients are diagnosed with ovarian cancer is 69 years, while in the present study, a median age of 66 years was observed in the German group. In the Romanian group the median age at which patients are diagnosed with ovarian cancer was 57 years.

According to the literature, ovarian cancer is diagnosed after menopause but also depends on ethnicity and geographical area. The majority of the ovarian cancer patients' age among the global population is between 60 and 64 years, with the typical age of 63 at diagnosis. Results of a Chinese study suggested that the Asian population is younger at diagnosis, with ovarian cancer being diagnosed in the majority of the studied cases at an age of 50 years (32).

The mean age of the patients in the German group was 66 years, which is over the globally reported age range, while in the Romanian group the mean age of the patients was 57 years, which was obviously younger than the globally reported parameters, but older than the parameters reported in the Chinese study.

Starting from this point, the disease dynamics and further development of the disease were assessed. A comparison between the two centers under observation regarding the stages of ovarian cancer was carried out. Although in stage 1 there were no significant differences regarding the comparison between the two centers, in stage 2 and afterwards stage 3 there were significant differences during the comparison between the two centers. The significant difference identified in stage 3 between the two countries suggests that medical information, education among this population, early diagnosis, patient monitoring, and prevention through medical treatment, may play a key role in disease dynamics. Prevention and regular medical check-ups play a very important role in decreasing mortality. This information was also mentioned in a population-based case-control study of ovarian cancer in women during the period 1971-2011 (33).

The results of the present study are sustained by the results of the study of global surveillance of cancer survival for the period 1995-2009 taken from 279 population-based registries in 67 countries. The present findings show that 
Table I. Descriptive statistics for our samples.

\begin{tabular}{lcccc}
\hline Sample & No. of patients $(\mathrm{n})$ & Mean age & Standard deviation & Standard error of the mean \\
\hline DE & 49 & 66.1 & 13.5 & 1.9 \\
RO & 97 & 57.3 & 13.0 & 1.3 \\
\hline
\end{tabular}

DE, German patient group; RO, Romanian patient group.

Table II. Comparison between tumor stages in the two countries.

\begin{tabular}{lccc}
\hline Stage & DE & RO & P-value \\
\hline I & $4.1 \%$ (2 subjects $)$ & $11.3 \%(11$ subjects $)$ & P $>0.05$ \\
II & $28.6 \%(14$ subjects $)$ & $5.2 \%$ (5 subjects $)$ & P $<0.001$ \\
III & $67.4 \%$ (33 subjects) & $83.5 \%$ (81 subjects $)$ & $P=0.044$ \\
\hline
\end{tabular}

$\mathrm{DE}$, German patient group; RO, Romanian patient group.

Table III. Risk analyses ${ }^{\mathrm{a}}$.

\begin{tabular}{|c|c|c|c|c|}
\hline $\mathrm{N}=32$ & Stage 2 & Stage 1 & Total & \\
\hline RO & 5 & 11 & 16 & $\mathrm{P}=0.0011$ \\
\hline $\mathrm{DE}$ & 14 & 2 & 16 & $\mathrm{OR}=0.06$ \\
\hline Total & 19 & 13 & 32 & $95 \% \mathrm{CI}=0.01-0.40$ \\
\hline $\mathrm{N}=133$ & Stage 3 & Stage 2 & Total & \\
\hline $\mathrm{RO}$ & 81 & 5 & 86 & $\mathrm{P}=0.0001$ \\
\hline DE & 33 & 14 & 47 & $\mathrm{OR}=6.87$ \\
\hline Total & 114 & 19 & 133 & $95 \% \mathrm{CI}=2.29-20.61$ \\
\hline $\mathrm{N}=146$ & Stage 3 & Stage $1+2$ & Total & \\
\hline RO & 81 & 16 & 97 & $P=0.026$ \\
\hline $\mathrm{DE}$ & 33 & 16 & 49 & $\mathrm{OR}=2.45$ \\
\hline Total & 114 & 32 & 146 & $95 \% \mathrm{CI}=1.10-5.47$ \\
\hline
\end{tabular}

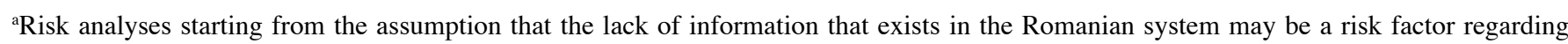
the development of new lethal diseases or a rapid increase from stage 1 to stage 3. DE, German patient group; RO, Romanian patient group. OR, odds ratio; CI, confidence interval.

worldwide surveillance of cancer survival is different and the differences are shown through access to early diagnosis and optimum treatment based on health policy and health-care systems (34).

In conclusion, the present results have shown the same pathology in different medical centers from different countries and underlines the different approach of ovarian cancer according to the medical system and according to the patients' willingness and knowledge to perform the necessary medical check-up at the appropriate moment in time. Thus, informing the patients, early diagnosis and standardized therapy play a key role in the medical act and varies from country to country.

\section{Acknowledgements}

Not applicable.

\section{Funding}

No funding was received.

\section{Availability of data and materials}

Further information concerning the present study is available from the corresponding author upon reasonable request. 


\section{Authors' contributions}

IMC, IS and AR conceived and planned the present study in detail. IMC and AEM extracted and analyzed the entire patient data. LM performed the computations and interpreted the patient data together with IMC, IS and AEM. IMC took the lead in writing the manuscript after LM and AEM wrote some parts of the manuscript with input from AR and IS. IMC wrote the manuscript in consultation with AR. AR and IMC supervised this study. All authors discussed the results and commented on the manuscript. All authors read and approved the final manuscript for publication.

\section{Ethics approval and consent to participate}

This study used pre-existing, anonymized and irreversibly de-identified data. Approval from the ethics committee was not required.

\section{Patient consent for publication}

Not applicable.

\section{Competing interests}

The authors declare that they have no competing interests.

\section{References}

1. Chen T, Jansen L, Gondos A, Emrich K, Holleczek B, Luttmann S, Waldmann A and Brenner H; Gekid cancer survival working group: Survival of cervical cancer patients in Germany in the early 21 st century: A period analysis by age, histology, and stage Acta Oncol 51: 915-921, 2012

2. McLemore MR, Miaskowski C, Aouizerat BE, Chen LM and Dodd MJ: Epidemiological and genetic factors associated with ovarian cancer. Cancer Nurs 32: 281-288, 2009.

3. Yoneda A, Lendorf ME, Couchman JR and Multhaupt HA: Breast and ovarian cancers: A survey and possible roles for the cell surface heparan sulfate proteoglycans. J Histochem Cytochem 60: 9-21, 2012.

4. Moghaddam SM, Amini A, Morris DL and Pourgholami MH: Significance of vascular endothelial growth factor in growth and peritoneal dissemination of ovarian cancer. Cancer Metastasis Rev 31: 143-162, 2012.

5. Saad AF, Hu W and Sood AK: Microenvironment and pathogenesis of epitelial ovarian cancer. Horm Cancer 1: 277-290, 2010.

6. Popa Z, Chiriac VD, Cobec IM, Lungeanu D, Craina M, Bernad E, Ionita I, Moleriu RD, Iacob D and Petre I: HPV cervical cancer screening. An analysis over HPV markers between worldwide statistics and Romanian reality. Rev Chim 68: 2459-2462, 2017.

7. Rosen DG, Yang G, Liu G, Mercado-Uribe I, Chang B, Xiao XS, Zheng J, Xue FX and Liu J: Ovarian cancer: Pathology, biology, and disease models. Front Biosci (Landmark Ed) 14: 2089-2102, 2009.

8. Kim A, Ueda Y, Naka T and Enomoto T: Therapeutic strategies in epithelial ovarian cancer. J Exp Clin Cancer Res 31: 14, 2012.

9. Lalwani N, Prasad SR, Vikram R, Shanbhogue AK, Huettner PC and Fasih N: Histologic, molecular, and cytogenetic features of ovarian cancers: Implications for diagnosis and treatment. Radiographics 31: 625-646, 2011.

10. Cho KR and Shih IM: Ovarian cancer. Annu Rev Pathol 4 : 287-313, 2009.

11. Barua A, Bitterman P, Abramowicz JS, Dirks AL, Bahr JM, Hales DB, Bradaric MJ, Edassery SL, Rotmensch J and Luborsky JL: Histopathology of ovarian tumors in laying hens: A preclinical model of human ovarian cancer. Int J Gynecol Cancer 19: 531-539, 2009.

12. Shih IM and Davidson B: Pathogenesis of ovarian cancer: Clues from selected overexpressed genes. Future Oncol 5: 1641-1657, 2009.
13. Karst AM and Drapkin R: Ovarian cancer pathogenesis: A model in evolution. J Oncol 2010: 932371, 2010.

14. Cobec IM, Sas I, Pirtea L, Cimpean AM, Moatar AE, Ceaușu RA and Raica M: Podoplanin as key player of tumor progression and lymph vessel proliferation in ovarian cancer. Anticancer Res 36: 5265-5272, 2016

15. Prat J: New insight into ovarian cancer pathology. Ann Oncol 23 (Suppl 10): S111-S117, 2012.

16. Murphy MA and Wentzensen N: Frequency of mismatch repair deficiency in ovarian cancer: A systematic review. Int J Cancer 129: 1914-1922, 2011.

17. Li J, Fadare O, Xiang L, Kong B and Zheng W: Ovarian serous carcinoma: Recent concepts on its origin and carcinogenesis. J Hematol Oncol 5: 8, 2012

18. Markert EK, Levine AJ and Vazquez A: Proliferation and tissue remodeling in cancer: The hallmarks revisited. Cell Death Dis 3: e397, 2012.

19. Kurman RJ and Shih IM: The origin and pathogenesis of epithelial ovarian cancer: A proposed unifying theory. Am J Surg Pathol 34: 433-443, 2010.

20. Kurman RJ and Shih IM: Molecular pathogenesis and extraovarian origin of epithelial ovarian cancer-shifting the paradigm. Hum Pathol 42: 918-931, 2011.

21. Vang R, Shih IM and Kurman RJ: Ovarian low-grade and high-grade serous carcinoma: Pathogenesis, clinicopathologic and molecular biologic features, diagnostic problems. Adv Anat Pathol 16: 267-282, 2009.

22. Tew WP, Colombo N, Ray-Coquard I, Oza A, del Campo J, Scambia G and Spriggs D: VEGF-trap for patients (pts) with recurrent platinum-resistant epithelial ovarian cancer (EOC): Preliminary results of a randomized, multicenter phase II study. J Clin Oncol 25 (Suppl 18): S5508, 2007.

23. Colombo N, Mangili G, Mammoliti S, Kalling M, Tholander B, Sternas L, Buzenet G and Chamberlain D: Aflibercept(VEGF Trap) for advanced epithelial ovarian cancer (EOC) patients (pts) with symptomatic malignant ascites: Preliminary results of a pilot study. J Clin Oncol 26 (Suppl 15): S14598, 2008.

24. Leamon CP, Lovejoy CD and Nguyen B: Patient selection and targeted treatment in the management of platinum-resistant ovarian cancer. Pharmgenomics Pers Med 6: 113-125, 2013.

25. Cimpean AM, Cobec IM, Ceaușu RA, Popescu R, Tudor A and Raica M: Platelet derived growth factor BB: A 'Must-have' therapeutic target 'Redivivus' in ovarian cancer. Cancer Genomics Proteomics 13: 511-517, 2016.

26. Vrdoljak E, Wojtukiewicz MZ, Pienkowski T, Bodoky G, Berzinec P, Finek J, Todorović V, Borojević N and Croitoru A; South Eastern European Research Oncology Group: Cancer epidemiology in Central, South and Eastern European countries. Croat Med J 52: 478-487, 2011.

27. Ferlay J, Steliarova-Foucher E, Lortet-Tieulent J, Rosso S, Coebergh JW, Comber H, Forman D and Bray F: Cancer incidence and mortality patterns in Europe: Estimates for 40 countries in 2012. Eur J Cancer 49: 1374-1403, 2013.

28. Zheng L, Cui C, Shi O, Lu X, Li YK, Wang W, Li Y and Wang Q: Incidence and mortality of ovarian cancer at the global, regional, and national levels, 1990-2017. Gynecol Oncol 159: 239-247, 2020.

29. Shazly SA,Laughlin-Tommaso SK, Dowdy SC and Famuyide AO: Staging for low malignant potential ovarian tumors: A global perspective. Am J Obstet Gynecol 215: 153-168, 2016.

30. Matz M, Coleman MP, Carreira H, Salmerón D, Chirlaque MD and Allemani C; CONCORD Working Group: Worldwide comparison of ovarian cancer survival: Histological group and stage at diagnosis (CONCORD-2). Gynecol Oncol 144: 396-404, 2017.

31. Warren JL, Harlan LC, Trimble EL, Stevens J, Grimes M and Cronin KA: Trends in the receipt of guideline care and survival for women with ovarian cancer: A population-based study. Gynecol Oncol 145: 486-492, 2017.

32. Shen F, Chen S, Gao Y, Dai X and Chen Q: The prevalence of malignant and borderline ovarian cancer in pre- and postmenopausal Chinese women. Oncotarget 8: 80589-80594, 2017.

33. Quaresma M, Coleman MP and Rachet B: 40-year trends in an index of survival for all cancers combined and survival adjusted for age and sex for each cancer in England and Wales, 1971-2011: A population-based study. Lancet 385: 1206-1218, 2015.

34. Allemani C, Weir HK, Carreira H, Harewood R, Spika D, Wang XS, Bannon F, Ahn JV, Johnson CJ, Bonaventure A, et al: Global surveillance of cancer survival 1995-2009: Analysis of individual data for $25,676,887$ patients from 279 population-based registries in 67 countries (CONCORD-2). Lancet 385: 977-1010, 2015 . 